\title{
The Effect of Ketoconazole on Amphotericin B in a Model of Disseminated Aspergillosis
}

\begin{abstract}
The potential of ketoconazole prophylaxis to antagonize the activity of amphotericin B against aspergilli was investigated in vitro and in neutropenic mice. Exposure of Aspergillus fumigatus (six strains) and of Aspergillus flavus or Aspergillus niger to ketoconazole resulted in a uniform increase of the minimal fungicidal activity of amphotericin $B$, from $0.15-0.63 \mathrm{mg} /$ liter to $>2.5 \mathrm{mg} /$ liter in a microwell assay. To test the relevance of this antagonism in vivo, we challenged neutropenic mice iv with a lethal dose of conidia from two strains of $A$. fumigatus and then treated the mice first with ketoconazole and then with amphotericin B or amphotericin B plus ketoconazole. Pretreatment with ketoconazole for $48 \mathrm{hr}$ completely abolished the protective effect of a subsequent therapy with amphotericin B, whether ketoconazole therapy was stopped $(P<.001)$ or not $(P<.001)$. Ketoconazole given alone had no significant effect on survival. Our data show that ketoconazole not only antagonized the fungicidal activity of amphotericin $B$ in vitro but also abolished in vivo the protective effect of the only drug shown to be useful in the therapy of aspergillosis. The clinical importance of this antagonism, which is not limited to Aspergilli in vitro, requires careful consideration before ketoconazole prophylaxis can be recommended for patients at high risk of developing invasive opportunistic fungal infections.
\end{abstract}

Patients with marked and prolonged neutropenia are at high risk of developing an opportunistic mycosis. Thus prevention of fungal infection has become a major concern in this population. At necropsy, $24 \%-64 \%$ of patients with acute myelogenous leukemia are found to have an invasive fungal infection [1-8]. In some centers [1-3] opportunistic yeasts, commonly species of Candida, prevail, whereas in others [6-8] aspergilli are the leading opportunistic fungal pathogens.

Efforts to prevent candidiasis or aspergillosis might differ markedly because the former commonly results from mucosal invasion after colonization [9] and the latter usually is a consequence of inhalation of spores [10]. Thus, suppression of mucosal colonization cannot be expected to prevent aspergillosis. Furthermore, aspergilli and species of Candida differ in their susceptibility to antimycotic agents; e.g., Aspergilli are less susceptible to imidazoles [11]. Thus

Received for publication August 16, 1984, and in revised form November 14, 1984.

This work was supported by grant no. 3.815.0.83 from the Swiss National Science Foundation and by the EMDO Foundation.

We thank Ursula Schärer for technical assistance.

Please address requests for reprints to Dr. A. Schaffner, Medizinische Klinik, Universitätsspital, CH-8091 Zürich, Switzerland. it is not surprising that in a study [12] comparing prophylaxis of fungal infections with ketoconazole or miconazole in patients undergoing intensive antitumor chemotherapy, aspergillosis was discovered in $24 \%$ of patients at necropsy.

Because imidazoles have the potential to antagonize the antifungal activity of amphotericin B [13-15] (the most effective drug for therapy of most invasive opportunistic mycoses, including aspergillosis $[16,17])$, we became interested in the question of whether ketoconazole prophylaxis that was recently advocated in neutropenic patients $[12,18,19]$ would influence the efficacy of a subsequent therapy of aspergillosis with amphotericin B. We therefore investigated the effects of ketoconazole on the susceptibility of aspergilli to amphotericin B in vitro and in vivo in neutropenic mice.

\section{Materials and Methods}

Organisms. Six clinical isolates of Aspergillus fumigatus, all from immunocompromised patients with invasive disease and all isolated at the University Hospital of Zürich, and a strain each of Aspergillus flavus and Aspergillus niger isolated from patients at the University Hospital in San Diego were propagated, and suspensions of single spores were prepared as previously described [10]. 
In vitro studies on the interaction of ketoconazole and amphotericin $B$. For in vitro use, ketoconazole (gift from Janssen Pharmaceutica, New Brunswick, NJ) and amphotericin B (pure crystalline form; gift from E. R. Squibb \& Sons, Princeton, $\mathrm{NJ}$ ) were dissolved in dimethylsulfoxide (DMSO) at a concentration of $1 \mathrm{mg} / \mathrm{ml}$ and were diluted to the desired concentration in a mixture of 90\% Sabouraud dextrose broth (Difco, Detroit) and $10 \% 0.15 M$ phosphate buffer (SAB-PB; pH 7.4).

The fungicidal test was begun by dispensing 30-40 conidia, suspended in $100 \mu \mathrm{l}$ of trypticase-soy broth (Difco), into 300- $\mu$ l-flat-bottom clusterwell plates (Nunk, Kamstrup, Denmark). Plates were incubated at room temperature until the transformation of spores into mycelia was completed (14-18 hr). Germination was verified for each well by phase-contrast microscopy. The plates were then centrifuged at $800 \mathrm{~g}$ for $15 \mathrm{~min}$. The supernatant was aspirated through a 23-gauge needle and replaced by ketoconazole in SAB-PB or in SAB-PB with the appropriate concentration of DMSO. After incubation at $37 \mathrm{C}$ for the indicated time periods, plates were again centrifuged and the supernatant replaced by serial dilutions of amphotericin B in SAB-PB or in SAB-PB mixed with DMSO and were incubated for $24 \mathrm{hr}$ at $37 \mathrm{C}$. Plates were then centrifuged again and the supernatant was replaced by trypticase-soy broth to promote fungal outgrowth. Plates were inspected daily for six days for fungal colonies, and one drop of $5 \%$ aqueous methylene blue was added to each well with fungal growth to prevent sporulation and, thus, cross contamination. Results of minimal fungicidal concentrations are given as the lowest concentration at which no fungal growth occurred within six days after removal of amphotericin B. With the exception of a rare skipwell, all wells were sterilized at this concentration, whereas all wells produced fungi at the next lower concentration. In initial experiments wells were washed one to three times with SAB-PB after the removal of ketoconazole or amphotericin $B$, but washing had no influence on the test results and was later omitted. Furthermore we initially inspected the plates for up to three weeks, but prolonged incubation did not produce more fungal colonies.

Animals. Throughout the study female NMRI mice (IVANOVAS, Kisslegg, Federal Republic of Germany) were used. Mice were housed for at least 10 days in our facility, and immunosuppression was not started before the mice had reached a weight of

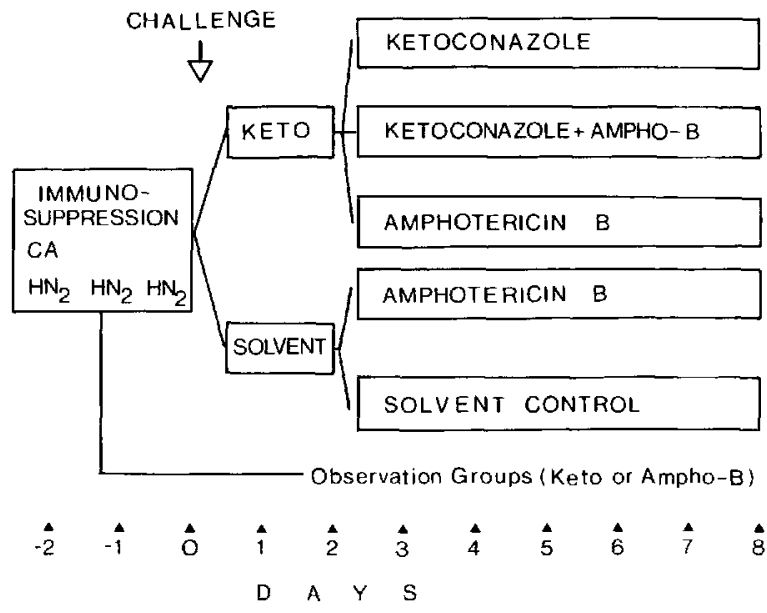

Figure 1. Diagram of experimental antimycotic regimens. Mice were immunosuppressed starting two days before challenge with cortisone acetate (CA) and nitrogen mustard $\left(\mathrm{HN}_{2}\right)$. Immediately after infection, mice received ketoconazole or solvent by gavage for $24-48 \mathrm{hr}$ followed by either ketoconazole, amphotericin B plus ketoconazole, amphotericin B, or solvents (carboxymethylcellulose by gavage, $5 \%$ glucose iv) for six days.

24-26 g. Cages were cleaned three times a week, and acidified water and food pellets were offered ad libitum.

Immunosuppression. To abolish the conidiacidal activity of macrophages, mice were given a single dose of $5 \mathrm{mg}$ of cortisone acetate (gift from Merck Sharp \& Dohme, West Point, Pa) sc [10]. Antimycelial defense was ablated by bone marrow suppression with nitrogen mustard (Merck Sharp \& Dohme) [10]. In preliminary experiments we found that iv injections (for three consecutive days) of $50 \mu \mathrm{g}$ of nitrogen mustard before infection gave an optimal neutropenic episode without spontaneous death and gave neutrophil counts $<100 / \mu l$ for the first four days after the anticipated challenge (figure 1) with a slow recovery of neutrophil counts over the next five days.

Challenge. Mice were challenged iv with conidia suspended in $0.5 \mathrm{ml}$ of $0.15 \mathrm{M} \mathrm{NaCl}$. Spores were quantitated by hematocytometer counts and culture of serial dilutions with the pour-plate technique [20]. In preliminary experiments we determined the $\mathrm{LD}_{50}$ and $\mathrm{LD}_{\mathbf{1 0 0}}$ of the two isolates of $A$. fumigatus that were selected for the in vivo studies in the immunosuppressed mice, as previously described [10]. For isolate no. 1 , the $\mathrm{LD}_{\mathrm{so}}$ was $8.4 \times 10^{2}$ and the $\mathrm{LD}_{100}$ was $4 \times 10^{4}$ spores; for isolate no. 4 , the $\mathrm{LD}_{50}$ was $7.3 \times 10^{2}$ and the $\mathrm{LD}_{100}$ was $3 \times 10^{4}$ spores. 
For in vivo studies of the protective effects of the antimycotic regimens, mice were challenged with an $\mathrm{LD}_{100}$.

Experimental antimycotic regimens. A dose of $2 \mathrm{mg}$ of amphotericin $\mathrm{B} / \mathrm{kg}$ per day was chosen on the basis of a comparison of the antifungal activity of plasma from groups of mice receiving graded doses of the drug for three days and the antifungal activity of plasma from two patients. To match the antifungal activity of plasma from two patients receiving 0.6 and $0.75 \mathrm{mg}$ of amphotericin $\mathrm{B} / \mathrm{kg}$ per day against $A$. fumigatus 1 in our microwell assay, we had to give mice $2 \mathrm{mg}$ of amphotericin $\mathrm{B} / \mathrm{kg}$ per day; undiluted plasma was fungicidal and, at a 1:2 or 1:4 dilution, fungistatic. After $1 \mathrm{mg} / \mathrm{kg}$ per day, undiluted plasma was only fungistatic and $0.5 \mathrm{mg} / \mathrm{kg}$ was inactive when compared with normal human or mouse plasma. A dose-range for ketoconazole was chosen on the basis of a comparison of the fungistatic activity of plasma obtained $2.5 \mathrm{hr}$ after the third daily dose of ketoconazole and the activity of mouse plasma to which known concentrations of the drug were added; the test organism we used was Trichophyton mentagrophytes (gift from Dr. A. Polak, Hoffmann La Roche, Basel, Switzerland). The test was modified by reducing the incubation temperature to $30 \mathrm{C}$, and the fungistatic activity was read at $48 \mathrm{hr}$. Plasma from mice receiving 150 or $200 \mathrm{mg}$ of ketoconazole per day was as active as plasma spiked with $3.5 \mathrm{mg}$ of ketoconazole/liter but was less active than plasma with $7 \mathrm{mg} /$ liter. A daily dose of $100 \mathrm{mg}$ of ketoconazole $/ \mathrm{kg}$ resulted in fungistatic activity identical to that of plasma to which $1.8 \mathrm{mg}$ of ketoconazole/liter was added but less active than plasma with $3.5 \mathrm{mg} /$ liter added. Thus a daily ketoconazole dose of $100-200 \mathrm{mg}$ in mice resulted in blood activities comparable to those of drug concentrations obtained in clinical therapy [21, 22].

The study design for the in vivo chemotherapeutic trials are shown in figure 1. Ketoconazole (100-200 $\mathrm{mg} / \mathrm{kg}$ per day) suspended in $0.2 \mathrm{ml}$ of carboxymethylcellulose with $0.01 \%$ Tween 80 was given by gavage. Amphotericin B-deoxycholate (Squibb), in a dose of $2 \mathrm{mg}$ of amphotericin $\mathrm{B} / \mathrm{kg}$ per day, was injected iv in a volume of $0.5 \mathrm{ml}$ of $5 \%$ glucose. For better tolerance the dose was divided into two injections that were given at intervals of 60-120 min.

Histology and quantitative cultures of organs. For histopathologic evaluation of lesions, organs were fixed in $10 \%$ phosphate-buffered formalin $(\mathrm{pH}$ 7.4) immediately after the killing of the animals, embedded in methacrylate, and stained by Giemsa's method. For quantitative cultures, individual organs were homogenized with Teflon pestles in $5 \mathrm{ml}$ of Sabouraud's broth [10] by using a hand drill (CSB 420-E; Scintilla AG, Solothurn, Switzerland) set at $400 \mathrm{rpm}$. Serial 1:10 dilutions of homogenates were mixed in pour plates with Sabouraud dextrose agar (Difco) and were read after $48 \mathrm{hr}$ at $37 \mathrm{C}$.

Evaluation of the protective effect of antimycotic regimens: statistical analysis. Survival of mice was recorded daily for at least 40 days after challenge. The $L D$ values were computed by the method of Reed and Munch [23]. Statistical significance of differences in survival were assessed by using $2 \times \mathrm{k}$ contingency tables with the Bonferroni- $\chi^{2}$ statistics ta-

Table 1. The susceptibility of aspergilli to amphotericin B after exposure to various concentrations of ketoconazole for $8 \mathrm{hr}$.

\begin{tabular}{|c|c|c|c|c|c|}
\hline \multirow[b]{2}{*}{ Fungus } & \multicolumn{5}{|c|}{ MFC after ketoconazole concentration (mg/liter) of* } \\
\hline & 0 & 0.5 & 1 & 2 & 4 \\
\hline A. fumigatus 1 & 0.63 & 2.5 & 5.0 & 5.0 & 5.0 \\
\hline A. fumigatus 2 & 0.31 & $\cdots$ & 5.0 & 5.0 & 5.0 \\
\hline A. fumigatus 3 & 0.31 & 1.25 & 5.0 & 5.0 & 5.0 \\
\hline A. fumigatus 4 & 0.31 & 1.25 & 2.5 & 2.5 & 5.0 \\
\hline A. fumigatus 5 & 0.15 & . & 1.25 & 2.5 & 5.0 \\
\hline A. fumigatus 6 & 0.63 & $\ldots$ & 5.0 & 5.0 & 5.0 \\
\hline mean & 0.38 & $\ldots$ & 3.5 & 3.75 & 5.0 \\
\hline A. niger & 0.15 & $\cdots$ & 2.5 & 2.5 & 2.5 \\
\hline A. flavus & 0.15 & $\ldots$ & 5.0 & 5.0 & $-\dagger$ \\
\hline
\end{tabular}

* MFC $=$ lowest concentration of amphotericin B (mg/liter) that sterilized microwells. Serial 1:2 dilutions from 10 to $0.07 \mathrm{mg}$ of amphotericin $\mathrm{B} /$ liter were tested in quadruplicate microwells.

$\uparrow$ At this concentration ketoconazole was fungicidal. 
Table 2. The influence of the duration and timing of ketoconazole exposure on the extent of antagonism to amphotericin $\mathrm{B}$.

\begin{tabular}{lcc}
\hline $\begin{array}{l}\text { Hr of } \\
\text { pretreatment }\end{array}$ & $\begin{array}{l}\text { Ketoconazole }+ \\
\text { amphotericin B }\end{array}$ & MFC (mg/liter) \\
\hline 0 & no & 0.31 \\
3 & no & 1.25 \\
6 & no & 2.5 \\
10 & no & 5.0 \\
0 & yes & 0.61 \\
3 & yes & 2.5 \\
10 & yes & 10.0 \\
\hline
\end{tabular}

NOTE. One milligram per liter of ketoconazole was added to microwells with hyphae from $A$. fumigatus 1 , at the indicated times, before centrifugation of the microplates and replacement of the culture medium by serial 1:2 dilutions of amphotericin B (0.15-10 mg/liter). The concentration of ketoconazole added with amphotericin B was also $1 \mathrm{mg} /$ liter. MFC = lowest concentration of amphotericin B that sterilized microwells.

bles for correction of multiple comparisons [24]. Differences between mean values were examined by Student's $t$ test [24].

\section{Results}

Effect of ketoconazole on the fungicidal activity of amphotericin $B$ in vitro. Pretreatment of six clinical isolates of $A$. fumigatus, or of a strain of $A$. flavus and of $A$. niger, with ketoconazole at concentrations corresponding to therapeutic drug levels [21, 22] uniformly suppressed the fungicidal activity of amphotericin B (table 1). Under the given conditions this antagonism was dose dependent, with a more pronounced effect at higher ketoconazole concentrations. On the other hand, prolonged incubation resulted in a more pronounced antagonism at a given concentration of ketoconazole. When ketoconazole was only added at the time when amphotericin B was given to the fungi, antagonism was minimal (table 2) but reproducible (data not shown). When, after preexposure, ketoconazole was again added with amphotericin $B$ to the cultures, the minimal fungicidal concentration of amphotericin B was further increased.

In vivo model of disseminated aspergillosis. Intravenous inoculation of an $\mathrm{LD}_{100}$ of spores resulted in a disseminated infection mainly affecting the liver $\left(1.3 \pm 0.8 \times 10^{3} \mathrm{cfu} / \mathrm{ml}\right.$ of organ homogenate on day 3 after challenge; mean \pm SD from five animals) and the kidneys $\left(7.3 \pm 0.6 \times 10^{2}\right)$ with only minimal infection of other organs, such as the brain (two out of five animals with positive culture; $10 \mathrm{cfu} / \mathrm{ml}$ of homogenate). Histopathologically, lesions consisted of anemic infarcts resulting from intravascular hyphal growth with thrombosis that was accompanied by mycelial invasion of the surrounding tissue. A cellular inflammatory reaction of the fungi was absent (figure 2). Death occurred within 12 days after challenge, mainly within the first eight days when neutrophil counts were drastically reduced.

Effects of ketoconazole pretreatment on the protective activity of amphotericin B in vivo. To evaluate whether our model of disseminated aspergillosis would be suited to investigating the effects of ketoconazole on the protective antifungal activity of amphotericin B, we challenged mice with $3 \times 10^{4}$ spores from strain no. 1 and then treated the mice for six days with either $200 \mathrm{mg}$ of ketoconazole $/ \mathrm{kg}$ per day or $2 \mathrm{mg}$ of amphotericin B/ $\mathrm{kg}$. All (16 of 16) control animals, which received the appropriate solvents, died within 10 days, whereas 15 of 16 mice receiving ketoconazole died in the same time period (ketoconazole vs. control, $P=.5$ ). In contrast, none of the 15 mice receiving amphotericin $B$ died within 40 days $(P<.001)$. Thus ketoconazole, even at high doses, did not prevent lethal infection, whereas amphotericin B started $12 \mathrm{hr}$ after infection was completely protective.

After these preliminary investigations we studied the effects of pretreating aspergillosis with ketoconazole on the subsequent protective activity of amphotericin B. In a first experiment, ketoconazole was started immediately after challenge and amphotericin B $24 \mathrm{hr}$ thereafter. As in the previous experiment, all control animals died, and amphotericin B protected the majority of mice. Seven of 13 mice receiving first ketoconazole and then amphotericin B plus ketoconazole, however, died $(P<.05)$. Again, ketoconazole alone was without effect (figure 3). In the next experiment, the time of pretreatment of aspergillosis was prolonged to $48 \mathrm{hr}$ and amphotericin B was started two days after challenge. While the delay in therapy reduced the protective effect of amphotericin B, antagonism of ketoconazole was even more marked. Furthermore, even when the administration of ketoconazole was stopped with the beginning of amphotericin B treatment, antagonism of the protective effect was still complete (figure 4). When the same experiment was repeated with another strain of $A$. fumigatus, comparable results were obtained (figure 5). Every experiment had control groups of at least eight mice receiving either 



Figure 2. Hepatic lesion four days after iv challenge with a lethal dose of conidia. Top: an hepatic vein filled with thrombotic material and neighbouring a large necrotic zone (clear area) with karyorrhexis and karyolysis $(\times 400)$. Bottom: at a higher magnification $(x 1,000)$, branching hyphae are seen in the thrombus with invasion of the contiguous tissue. Note the complete absence of a phagocytic inflammatory response to the hyphae. ketoconazole or amphotericin B, but no challenge, in order to monitor deaths unrelated to fungal infection. None of these animals died.

Finally, we investigated whether antagonism of the antifungal activity of amphotericin $B$ would also be reflected by differences in cfu in organ homogenates obtained from animals of the different therapeutic groups. Because the liver was the most heavily infected organ, results of quantitative cultures of the livers are shown (figure 6). Less homogenous, but still significant, differences reflecting ketoconazole antagonism of amphotericin B were obtained from cultures of the kidneys (results not shown).

\section{Discussion}

Opportunistic fungal infections have emerged as a major threat to patients undergoing intensive chemotherapy for malignancies such as acute myelogenous leukemia [1-8]. Thus the question of prevention of fungal infection in such patients has become an important concern. Removal of fungal spores from the patients' environment by filtration of the air is probably successful in reducing the incidence of infections with molds [25] but requires expensive equipment and cannot be expected to reduce the incidence of "endogenous" infections with 


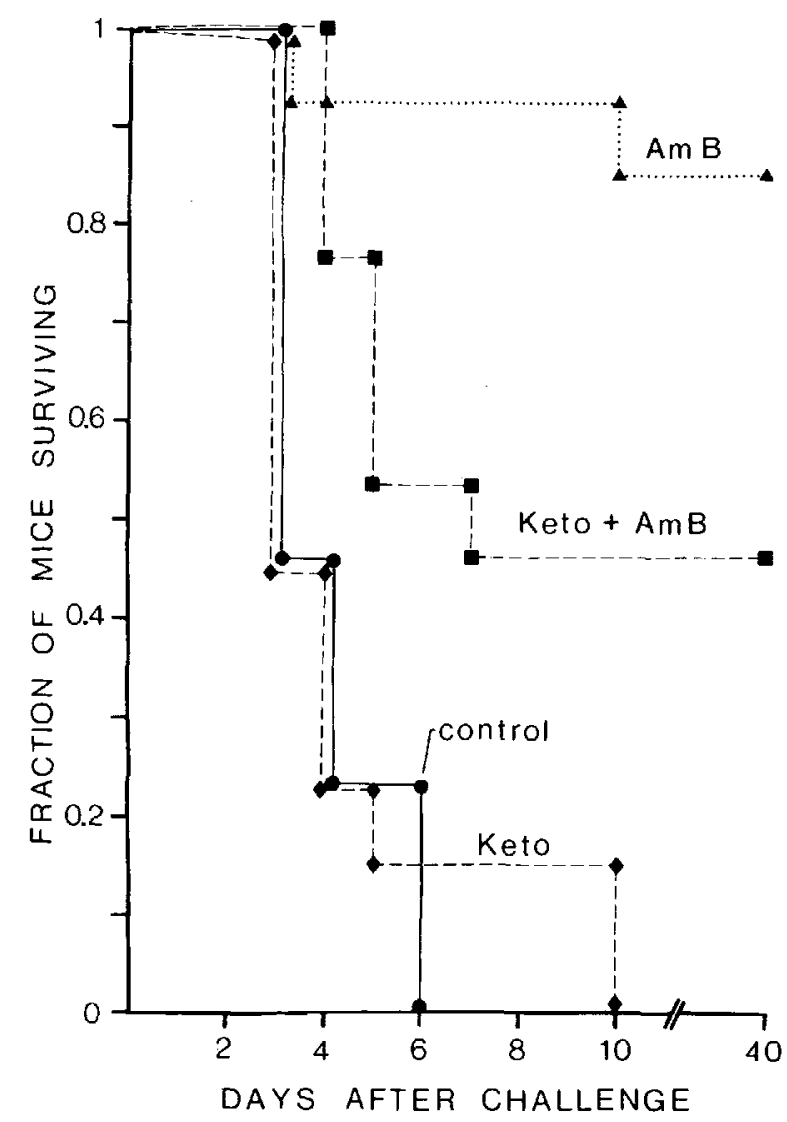

Figure 3. Antagonism of the protective activity of amphotericin B by ketoconazole. The figure shows the survival of mice ( $n=13$ in each group) that were challenged with $4.2 \times 10^{4} \mathrm{cfu}$ of $A$. fumigatus 1 . Ketoconazole (100 $\mathrm{mg} / \mathrm{kg}$ per day) was given for eight days (starting immediately after challenge) and amphotericin B ( $2 \mathrm{mg} / \mathrm{kg}$ per day) was given for six days (starting $24 \mathrm{hr}$ after challenge). Amphotericin B vs. control, $P<.01$; vs. ketoconazole, $P<.01$; vs. ketoconazole plus amphotericin $\mathrm{B}, P<.05$ (Bonferroni- $\chi^{2}$ test corrected for multiple comparisons).

yeasts. Thus two different approaches have been proposed to study the problem of opportunistic fungal infections in cancer patients; on one hand, chemoprophylaxis with ketoconazole $[12,18,19]$ - an oral systemic antifungal agent with few side effects and on the other hand, in bacterial infections, early empirical therapy with amphotericin B in neutropenic patients with febrile episodes not responding to antibacterial agents $[1,26]$.

Ketoconazole, like other imidazoles, has the potential to antagonize the antifungal activity of amphotericin $B$ in vitro [13-15], possibly by inhibiting ergosterol synthesis and thus depriving amphotericin $B$ of its binding site on the fungal cell membrane

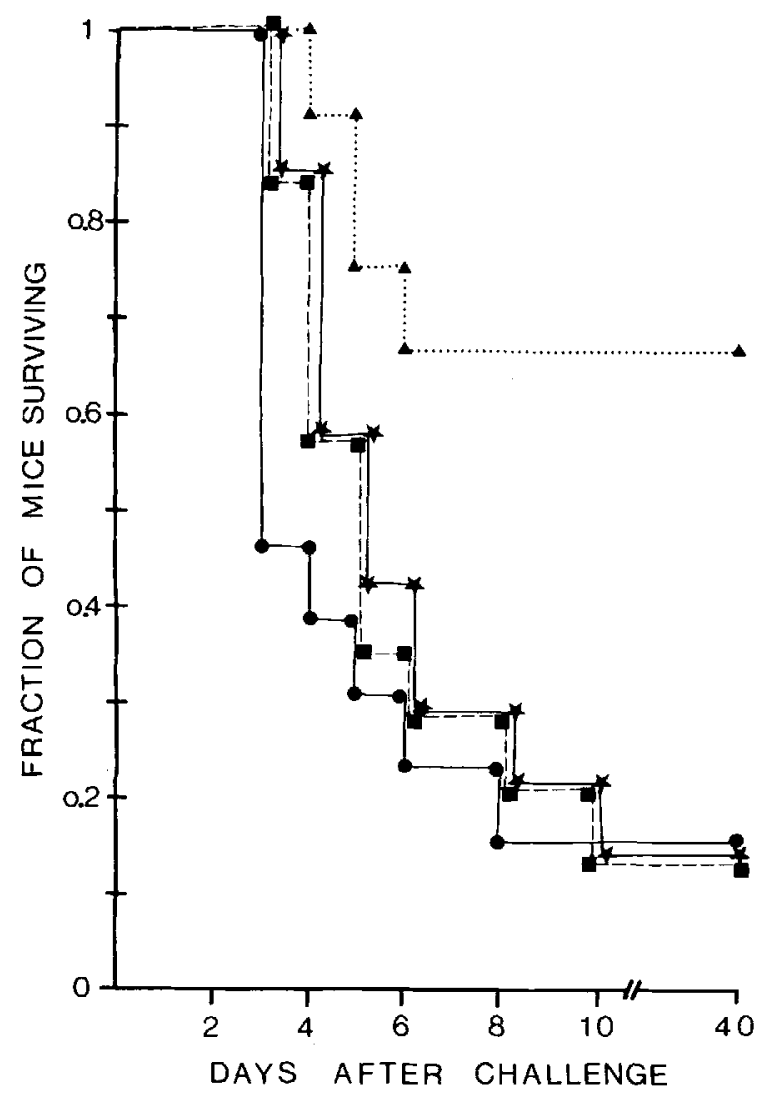

Figure 4. Abolishment of the protective activity of amphotericin $\mathbf{B}$ by ketoconazole. The figure shows the survival of mice ( $n=12-14$ in each group) challenged with $2.4 \times 10^{4} \mathrm{cfu}$ of $A$. fumigatus 1 . Ketoconazole (100 $\mathrm{mg} / \mathrm{kg}$ per day) was again started immediately after challenge and either stopped after $48 \mathrm{hr}$, when therapy with amphotericin B for six days was begun, or continued throughout therapy with amphotericin $\mathbf{B}$. = control; = amphotericin B plus ketoconazole; $\star=$ amphotericin B after ketoconazole; $\boldsymbol{A}=$ amphotericin B only. Amphotericin B vs. both groups receiving ketoconazole combined, $P=.0019$ (Fischer's exact test); amphotericin B alone vs. each other group, $P<.025$ (Bonferroni$\chi^{2}$ test corrected for multiple comparisons).

[15]. The in vitro interaction between imidazoles and amphotericin $B$ appears, however, to depend on the experimental design. The sequence of exposure to the two drugs appears to be of particular importance. It has been shown with Candida albicans and miconazole that antagonism is more marked when the fungal cells are first exposed to the imidazole and then exposed to amphotericin B, as compared with the reversed sequence [14]. This observation is supported by our finding that pretreatment with ketoconazole increased the minimal fungicidal ac- 


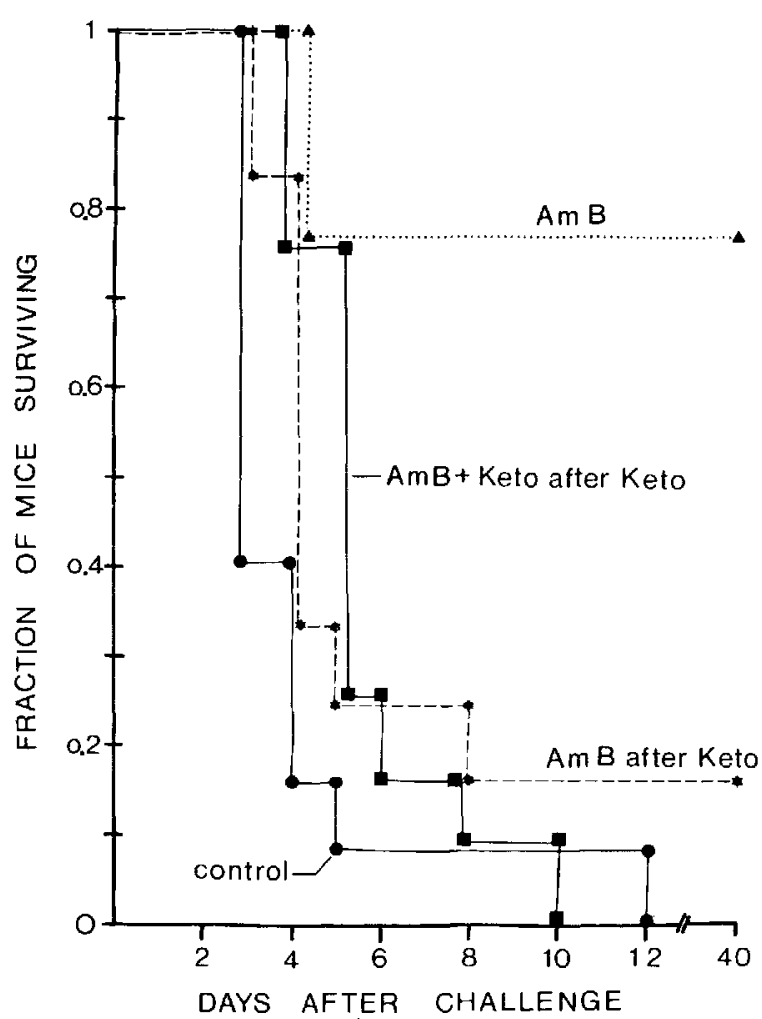

Figure 5. Abolishment of the protective activity of amphotericin B by ketoconazole. The figure shows the survival of mice ( $n=12$ in each group) challenged with $3.5 \times 10^{4} \mathrm{cfu}$ of $A$. fumigatus 4 . This experiment is identical to the experiment in figure 4 except that the ketoconazole dose was $150 \mathrm{mg} / \mathrm{kg}$ per day. Amphotericin $B$ vs. both groups receiving ketoconazole, $P=.0006$ (Fischer's exact test); amphotericin B alone vs. amphotericin $\mathrm{B}$ after ketoconazole, $P<.05$; vs. the two other groups, $P<.01$ (Bonferroni- $\chi^{2}$ test corrected for multiple comparisons).

tivity of amphotericin B much more than did simultaneous exposure (table 2). This observation appears logical if inhibition of ergosterol synthesis by the imidazole is assumed to be responsible for the interaction; antagonism would only be expected to be effective after the sterol composition of the fungal cell membrane had been altered. In contrast to antagonism, synergism was found in experiments in which fungi were exposed over several days to a combination of amphotericin B with an imidazole [14, 27]. Interpretation of these studies is difficult, however, because amphotericin B is not stable in vitro [28], and no attempts were made to replace the activity lost due to prolonged incubation. Thus it appears that these experiments might reflect the sequential activity of the two drugs rather than an interaction: amphotericin B being more active at first, when the ergosterol composition of the cell membrane is still unaffected, and the imidazole being still active several days after amphotericin B has been inactivated by oxidation. In any event, these discrepancies show that the clinical relevance of the in vitro interaction of imidazoles with amphotericin B can only be appreciated by in vivo experiments corresponding to clinical therapy.

Our studies of the effects of ketoconazole on the efficacy of subsequent therapy with amphotericin $B$ in a model of disseminated aspergillosis clearly document that the antagonism of the fungicidal activity of amphotericin B by prior exposure to ketoconazole is operative in vivo. In accordance with the in vitro studies, pretreatment with ketoconazole

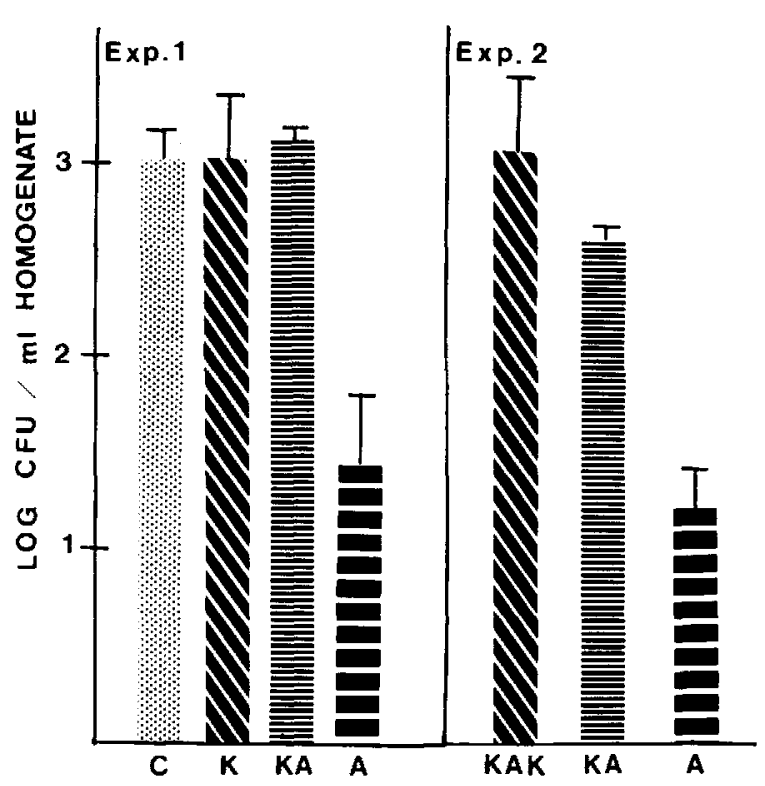

Figure 6. Comparison of $\mathrm{cfu}$ in liver homogenates of mice treated with different antimycotic regimens. In experiment 1 mice were challenged with an $L D_{100}$ of $A$. fumigatus 1 and killed $8 \mathrm{hr}$ after the first dose of amphotericin B. In experiment 2 mice were challenged with an $\mathrm{LD}_{100}$ of $A$. fumigatus 4 and killed $8 \mathrm{hr}$ after the second dose of amphotericin B. $\mathrm{C}=\mathrm{cfu}$ from control mice that did not receive antifungal drugs; $K=\mathrm{cfu}$ from mice that received three daily doses of ketoconazole $(100 \mathrm{mg}$ / $\mathrm{kg}) ; \mathrm{KA}=\mathrm{cfu}$ from mice that received ketoconazole (100 $\mathrm{mg} / \mathrm{kg}$ per day) plus amphotericin B ( $\mathrm{mg} / \mathrm{kg}$ per day) for two days; $K A K=\mathrm{cfu}$ from mice that received first ketoconazole and then ketoconazole plus amphotericin B (same doses as above). A vs. KA in each experiment, $P<.01$; A vs. C, $P<.01$; A vs. KAK, $P<.01$; KA vs. KAK, not significant; $K$ vs. $C$, not significant two-tailed Student's $t$ test). 
prevented the fungicidal activity of amphotericin B in vivo, as shown by quantitative cultures of liver homogenates (figure 6 ) and completely abolished the protective effect of amphotericin B (figures 4 and 5). The extent of the antagonism in the animal model suggests that the interaction might also be clinically relevant.

Antagonism of amphotericin B by ketoconazole has been shown previously in murine aspergillosis, but the effect was not dramatic. The results of these investigations are, however, difficult to compare with our study because the mice were not immunosuppressed, the drugs were given simultaneously, and only a fraction of our amphotericin $B$ dose was given to the animals [29]. Starting from the question of whether ketoconazole prophylaxis would interfere with subsequent therapy of a fungal breakthrough infection, we selected experimental conditions that would permit extrapolation to the clinical problem. We chose, therefore, a model of aspergillosis in which elimination of phagocytic defense resulted in histopathologic lesions comparable to clinical disease [12] and selected doses of antimycotic drugs corresponded to clinical therapy.

The efficacy of ketoconazole prophylaxis for prevention of invasive opportunistic mycosis in patients at high risk is not established. In a small but careful study, Brincker [19] found that $400 \mathrm{mg}$ of ketoconazole significantly reduced the incidence of oral thrush in cancer patients but that two of 19 patients treated with ketoconazole (a fraction identical to that of the placebo group) developed systemic candidiasis. Both patients in the ketoconazole group succumed to the infection in spite of therapy with amphotericin B and 5-flucytosine, whereas the two patients in the placebo group were cured. This observation might not be fortuitous because antagonism of amphotericin B by ketoconazole is as marked for C. albicans as it is for Aspergilli (A. S., unpublished observations) [15]. A recent study from England [18] compared the efficacy of ketoconazole with oral amphotericin $B$ and nystatin and suggested there was a reduction in the incidence of fungal infection in high-risk patients receiving ketoconazole. This study fails, however, to provide data that permit discrimination between superficial fungal infections, such as thrush, and disseminated opportunistic mycoses. Other studies show a high rate of breakthrough infection under ketoconazole prophylaxis $[12,30]$ in addition to a high-failure rate of amphotericin B in such patients [12].
In consideration of the available clinical experience with ketoconazole prophylaxis, our data $(I)$ show that ketoconazole might significantly interfere with subsequent therapy of aspergillosis with amphotericin $\mathrm{B}$, making this form of prophylaxis unsuitable for centers in which aspergilli are important pathogens; (2) indicate that ketoconazole prophylaxis, which has few side effects on the patient, might have an important, undesired effect of reducing the susceptibility of fungi to the only antimycotic agent shown to be effective in most invasive opportunistic mycoses; and (3) point out that future clinical evaluation of imidazoles for fungal prophylaxis should include analysis of the efficacy of amphotericin B therapy in patients with breakthrough mycosis.

\section{References}

1. Pizzo PA, Robichaud KJ, Gill FA, Witebsky FG. Empiric antibiotic and antifungal therapy for cancer patients with prolonged fever and granulocytopenia. Am J Med 1982;72:101-11

2. Edwards JE Jr, Lehrer RI, Stiehm ER, Fischer TJ, Young LS. Severe candidal infections. Clinical perspective, immune defense mechanisms and current concept of therapy. Ann Intern Med 1978;89:91-106

3. Wingard JR, Merz WG, Saral R. Candida tropicalis: A major pathogen in immunocompromised patients. Ann Intern Med 1979;91:539-43

4. Krick JA, Remington JS. Opportunistic invasive fungal infections in patients with leukaemia and lymphoma. Clin Haematol 1976;5:249-310

5. Young RC, Bennett JE, Vogel CL, Carbone PP, DeVita VT. Aspergillosis. The spectrum of the disease in 98 patients. Medicine 1970;49:147-73

6. Fraser DW, Ward JI, Ajello L, Plikaytis BD. Aspergillosis and other systemic mycoses: the growing problem. JAMA 1979;242:1631-5

7. Gerson SL, Talbot GH, Hurwitz S, Strom BL, Lusk EJ, Cassileth PA. Prolonged granulocytopenia: the major risk factor for invasive pulmonary aspergillosis in patients with acute leukemia. Ann Intern Med 1984;100:345-51

8. Stahel RA, Vogt P, Schüler G, Rüttner JR, Frick P, Oelz O. Systemic fungal infections in haematological malignancies: a growing problem. J Infect 1982;5:269-75

9. Stone HH, Kolb LD, Currie CA, Geheber CE, Cuzzell JZ. Candida sepsis: pathogenesis and principles of treatment. Ann Surg 1974;179:697-713

10. Schaffner A, Douglas H, Braude A. Selective protection against conidia by mononuclear and against mycelia by polymorphonuclear phagocytes in resistance to aspergillus. J Clin Invest 1982;69:617-31

11. Levine HB. Ketoconazole in the management of fungal disease. New York: ADIS Press, 1982

12. Meunier-Carpentier F, Cruciani M, Klastersky J. Oral prophylaxis with miconazole or ketoconazole of invasive fungal disease in neutropenic cancer patients. Eur J Cancer Clin Oncol 1983;19:43-8 
13. Schacter LP, Owellen RJ, Rathbun HK, Buchanan B. Antagonism between miconazole and amphotericin $B$ [letter]. Lancet 1976;2:318

14. Brajtburg J, Kobayashi D, Medoff G, Kobayashi GS. Antifungal action of amphotericin B in combination with other polyene or imidazole antibiotics. J Infect Dis 1982;146: $138-46$

15. Sud 1J, Feingold DS. Effect of ketoconazole on the fungicidal action of amphotericin B in Candida albicans. Antimicrob Agents Chemother 1983;23:185-7

16. Medoff G, Kobayashi GS. Strategies in the treatment of systemic fungal infections. N Engl J Med 1980;302:145-55

17. Utz JP. Chemotherapy of the systemic mycoses. Med Clin North Am 1982;66:221-33

18. Hann IM, Corringham R, Keaney M, Noone P, Fox J, Szawatkowski M, Prentice HG, Blacklock HA, Shannon M, Gascoigne E, Boesen E, Hoffbrand AV. Ketoconazole versus nystatin plus amphotericin $B$ for fungal prophylaxis in severely immunocompromised patients. Lancet 1982;1:826-9

19. Brincker $\mathbf{H}$. Prevention of mycosis in granulocytopenic patients with prophylactic ketoconazole treatment. Mykosen 1983;26:242-7

20. Schaffner A, Douglas H, Braude AI, Davis CE. Killing of Aspergillus spores depends on the anatomical source of the macrophage. Infect Immun 1983;42:1109-15

21. Brass C, Galgiani JN, Blascke TF, Defelice R, O'Reilly RA, Stevens DA. Disposition of ketoconazole, an oral antifungal in humans. Antimicrob Agents Chemother 1982; 21:151-8

22. Ginsburg CM, McCracken GH Jr, Olsen K. Pharmacology of ketoconazole suspension in infants and children. Antimicrob Agents Chemother 1983;23:787-9

23. Reed LJ, Muench $H$. A simple method of estimating fifty percent endpoints. American Journal of Hygiene 1938;27:493-7

24. Sachs L. Angewandte Statistik. Berlin: Springer, 1978

25. Schimpff SC, Young VM. Epidemiology and prevention of infection in the compromised host. In: Rubin RH, Young LS, eds. Clinical approach to infection in the compromised host. New York: Plenum, 1981:5-33

26. Stein RS, Kayser J, Flexner JM. Clinical value of empirical amphotericin B in patients with acute myelogenous leukemia. Cancer 1982;50:2247-51

27. Smith D, McFadden HW Jr, Miller NG. Effect of ketoconazole and amphotericin $B$ on encapsulated and non-encapsulated strains of Cryptococcus neoformans. Antimicrob Agents Chemother 1983;24:851-5

28. Cheung SC, Medoff G, Schlessinger D, Kobayashi GS. Stability of amphotericin B in fungal culture media. Antimicrob Agents Chemother 1975;8:426-8

29. Polak A, Scholer HJ, Wall M. Combination therapy of experimental candidiasis, cryptococcosis and aspergillosis in mice. Chemotherapy 1982;28:461-79

30. Acuna G, Winston DJ, Young LS. Ketoconazole prophylaxis of fungal infections in the granulocytopenic patient: a double blind randomized controlled trial [abstract no. 852]. In: Programs and abstracts of the 21st Interscience Conference on Antimicrobial Agents and Chemotherapy. Washington, DC: American Society for Microbiology, 1981 